\title{
Habilidades socioemocionales en las instituciones educativas
}

\author{
Socio-emotional skills in educational institutions
}

\author{
Moiria Noemí Cabanillas Tello (D) \\ Subdirectora I.E 16024-Huabal, Magister en Psicología Educativa. \\ Estudiante Doctorado Universidad César Vallejo, Perú
}

Rosangella del Jesús Rivadeneyra Pérez (D)

Estudiante Doctorado Universidad César Vallejo, Perú

Celia Ysabel Palacios Alva (D)

Dirección académica Personal de gestión y apoyo institucional de USAT.

Magíster en educación con mención gestión y docencia educativa.

Estudiante Doctorado Universidad César Vallejo, Perú

\section{Bertila Hernández Fernández 10}

Dra. En Educación; Dra. en Gestión Pública y Gobernabilidad. Universidad César Vallejo, Filial Chiclayo - Perú

Autor para correspondencia: Moiria Noemí Gabanillas Tello

Correo: mcabanillast@ucvvirtual.edu.pe

\section{RESUMEN}

Las políticas educativas para afrontar esta sociedad global, digital y compleja en la actualidad vienen configurando e implementando nuevos modelos de formación básica en las escuelas, entre los que destacan el enfoque por competencias y la educación emocional. En este contexto, se realizó una revisión descriptiva de artículos científicos relacionados con las habilidades socioemocionales en las instituciones educativas. Las fuentes seleccionadas fueron quince, aprobadas entre los años 2016 - 2020, e indexadas a revistas publicadas en la base de datos: Dialnet, Redalyc, Google Scholar y SciELO. Los contenidos temáticos asociados a la variable de estudio expresan congruencia con la actuación docente, tareas colaborativas, gestión del aprendizaje, creencias, la danza, el apego, así como la aplicación y evaluación de programas de intervención. Las investigaciones examinadas presentan metodologías cualitativas y cuantitativas, con diseños etnográficos, biográficos, correlacional, descriptivos e interpretativos, estudio de casos; y en el proceso de recolección de datos utilizaron la entrevista, 
observación, grupos focales y de discusión, análisis de contenido, inventarios, escalas, test y cuestionarios, administrados a maestros y estudiantes. Los resultados de los documentos consultados corroboran que existe la necesidad de articular y transitar de una educación centrada en lo cognitivo hacia el desarrollo de competencias socioemocionales en los diversos actores y niveles educativos.

Palabras clave: competencias emocionales, habilidades socioemocionales, inteligencia emocional, educación.

\section{ABSTRACT}

The educational policies to face this global, digital and complex society are currently configuring and implementing new models of basic training in schools, among which the competencies approach and emotional education stand out. In this context, a descriptive review of scientific articles related to socio-emotional skills in educational institutions was carried out. The selected sources were 15, approved between the years 2016 - 2020, and indexed to journals published in the database: Dialnet, Redalyc, Google Scholar and SciELO. The thematic contents associated with the study variable express congruence with the teaching performance, collaborative tasks, learning management, beliefs, dance, attachment, as well as the application and evaluation of intervention programs. The researchers examined present qualitative and quantitative methodologies, with ethnographic, biographical, correlational, descriptive and interpretive designs, case studies; and in the data collection process they used the interview, observation, focus and discussion groups, content analysis, inventories, scales, tests and questionnaires, administered to teachers and students. The results of the consulted documents corroborate that there is a need to articulate and move from an education focused on the cognitive to the development of socio-emotional competencies in the various actors and educational levels.

Keywords: Emotional competences, socio-emotional skills, emotional intelligence, education.

\section{Introducción}

El modelo educativo que rige y orienta los procesos pedagógicos y propósitos de aprendizaje en las instituciones educativas nacionales está centrado en el desarrollo de competencias, facultades que se pretenden alcanzar a través de capacidades definidas como recursos que abordan conocimientos, actitudes y habilidades. Desde su implementación ha recibido numerosos cuestionamientos por su complejidad metodológica, escasa comprensión para combinar y evaluar los recursos antes mencionados, además porque sigue subrayando la importancia de las operaciones cognitivas para la construcción de aprendizajes; asimismo, los valores, actitudes y habilidades siguen subordinadas o desplazadas a ser trabajadas sin ningún criterio pedagógico. Esta aseveración se puede confrontar con lo presentando en el Diseño Curricular Nacional (2017) donde de manera literal solo se menciona a las habilidades socioemocionales (HSE) dentro de la conceptualización de competencia y en el marco del perfil relacionado con la gestión de proyectos emprendedores. Sucede lo mismo con las habilidades sociales aludidas únicamente en el enfoque encaminado a la búsqueda de la excelencia, es más, asume la habilidad como aptitud. (Ministerio de Educación 2017,17-27, 29-30).

La formación integral de los estudiantes necesita de la cohesión, articulación y complementariedad de la educación emocional y cognitiva, para ello es necesario que los docentes asocien el dominio disciplinar con la promoción de la inteligencia emocional, Vaello (2009) considera que un maestro emocionalmente competente puede subsanar en sus estudiantes aspectos que afectan el aprendizaje como la baja autoestima, irresponsabilidad, desmotivación, conformismo, vulnerabilidad y crear un clima acogedor y lograr un confortable proceso de enseñanza (p.11). Asimismo, los centros educativos deben constituirse en espacios emocionalmente inteligentes (Royo, 
2013). Por tanto, es primordial que las instituciones sean entornos agradables, afectivos e inclusivos para la construcción de una convivencia sana y emocionalmente armónica (Del Rey y Ortega 2001).

Esta investigación busca comprender y reflexionar críticamente las razones que están alterando el clima organizacional en las instituciones educativas evidenciando ruptura de relaciones interpersonales, actuaciones conflictivas entre docentes, escaso trabajo colaborativo e interdependiente, dificultades para regular y controlar sus emociones, así como manejo inapropiado del soporte socioemocional de los estudiantes. En este contexto, es necesario abordar las siguientes preguntas: ¿Qué estudios sobre el desarrollo de habilidades socioemocionales en las instituciones educativas se han publicado entre los años 2016 - 2020? ¿Qué HSE se promueven en las instituciones educativas? y ¿Cuál son los aportes de las HSE a las instituciones educativas?

Rubiales et al., (2018), revisan y analizan el impacto de programas de entrenamiento socioemocional aplicados durante el 2011 y 2015 a niños, niñas y adolescentes; determinando que, las intervenciones realizadas han tenido incidencia y efectividad positiva en el desarrollo de la competencia e inteligencia emocional, reducción del estrés y la agresividad; fortalecimiento de la salud mental, los niveles de autoestima y autoeficacia; asimismo, contribuyeron en potenciar la socialización grupal. Del mismo modo, la revisión teórica que realiza García et al., (2019) sobre el apego entre docentes y estudiantes en revistas indexadas y publicadas entre el 2015 y 2019, sintetizan que, la relación afectiva, sensible y cálida, docente - alumnos, permite la protección y seguridad emocional en los niños; repercute en los logros de aprendizaje, el desarrollo de habilidades sociales, regulación de conductas y ajuste emocional; confirman que este acercamiento recíproco mitiga el acoso, la agresividad interpersonal, y favorece la construcción de un clima de aula basado en lazos de amistad saludables, mejor comprensión y escucha entre las partes, y mejora el desempeño en la enseñanza de los maestros.

Las tendencias actuales demandan que las organizaciones educativas incluyan en sus proyectos educativos competencias socioemocionales con el propósito de formar estudiantes capaces de enfrentar desafíos y logren consolidar un proyecto de vida trascendente. Estas competencias de acuerdo con Goleman (1995), Scott (2015) y Romero $(2016,9)$ se caracterizan por ser personales, de autorregulación, sociales y de aprendizaje. Las primeras tienen la intención de alcanzar el conocimiento personal, gestionar las emociones, asumir riesgos, estimular la iniciativa, creatividad, responsabilidad, motivación y la autoconciencia. Las de autorregulación están conectadas a confiabilidad, integridad, adaptabilidad e innovación. Las sociales se manifiestan en la empatía, compasión, trabajo en equipo, resolución de desencuentros conflictivos, comunicación, flexibilidad, reciprocidad y aprovechamiento positivo de las adversidad y diversidad. Y las de aprendizaje, relacionadas con habilidades para la gestión, superar dificultades, y transformar los errores y fracasos en oportunidades. Todas estas competencias contribuyen a la cohesión sinérgica de la razón y la emoción; por tanto, las personas se pueden desenvolver en diversos campos en forma competitiva y eficiente.

Barrientos (2016), conceptualiza las HSE como "el conjunto de competencias y habilidades que aseguran el manejo y regulación de los estados de ánimo y los sentimientos, conllevando a modelar comportamientos emocionales y sociales 
coherentes en el contexto donde se desenvuelven", y West (2016) identifica a la "perseverancia, sociabilidad y curiosidad" como habilidades que se encuentran conexas a la HSE, y son responsables de motivar, energizar y dirigir tanto el pensamiento y las conductas". A su vez las competencias o inteligencia socioemocional está referida a habilidades y actitudes que facilitan la comprensión, expresión y regulación oportuna y adecuada de manifestaciones emocionales positivas, negativas y destructivas, incluyen también capacidades y aprendizaje que se obtienen mediante la experiencia y se denotan en un escenario determinado. Su percepción tangible se visualiza en las interacciones de las personas con su ambiente. Por ende, su desarrollo trae consigo la adaptación de las personas a diversos contextos, superación de situaciones difíciles de la vida, mejora de los procesos de aprendizaje, desempeños eficientes y de calidad, éxito profesional, efectividad en la solución de problemas, bienestar psicosocial, armonía en la salud mental, física y emocional. (Rubiales et al., 2018, 168 -167)

\section{Método}

El proceso metodológico que guió la revisión de los artículos científicos se relaciona con el tipo de investigación cuantitativa de carácter exploratoria - bibliométrica, y el diseño que se trabajó fue el descriptivo. Los métodos teóricos empleados fueron: el histórico - lógico pues se tuvo en cuenta la secuencialidad y progresión de los estudios comprendidos entre el 2016 y 2020; y el análisis - síntesis, que posibilitaron descomponer, relacionar, combinar y sintetizar aspectos esenciales de la información registrada. (Cerezal y Fiallo 2002, 51 - 57). En relación con la muestra de estudio, se optó por elegir 15 documentos indexa- dos, se utilizó para ello el muestreo no probabilístico intencional y por conveniencia, debido que se direccionó la indagación según el interés de las investigadoras (Otzen y Manterola 2017, 230). Además, la técnica utilizada comprendió el análisis documental y como instrumento se administró una matriz de marco lógico.

La exploración se realizó mediante una planificación intencionada que parte de la elección de la temática a investigar; la definición de los propósitos, seguido de la organización del portafolio virtual para almacenar, ordenar y codificar los archivos que guardaron similitud con el objeto de estudio. Luego se procedió a la búsqueda de información siguiendo procedimientos que agrupan varias etapas con actividades interactivas lógicas. La primera etapa consistió en gestionar recursos educativos en plataformas educativas digitales para identificar información disponible sobre HSE logrando seleccionar e ingresar a 4 bibliotecas web (Dialnet, Redalyc, Google Scholar y SciELO); en la segunda etapa se identificó de manera precisa datos biográficos de cada documento priorizado. Y, en la tercera etapa se realizó la trascripción de los aspectos estructurales básicos y sustanciales de los aportes investigativos. 


\title{
III. Resultados
}

\author{
Tabla 1
}

Descriptores bibliográficos: Identidad de los artículos.

\begin{tabular}{|c|c|c|}
\hline Autor (es) y año & Título & Revista indexada \\
\hline $\begin{array}{l}\text { Aragó, A., Zorrilla, L. } \\
\text { y Balaguer, P. (2016) }\end{array}$ & $\begin{array}{l}\text { Cómo trabajar las HSE a través de la danza } \\
\text { y su importancia en la escuela. }\end{array}$ & $\begin{array}{l}\text { Quaderns digitals: Revista de } \\
\text { Nuevas Tecnologías y Socie- } \\
\text { dad. }\end{array}$ \\
\hline Jakiwara, L. G. (2016) & $\begin{array}{l}\text { Las HSE en los jóvenes: una propuesta de } \\
\text { desarrollo humano integral. }\end{array}$ & $\begin{array}{l}\text { Revista de Ciencias de la Co- } \\
\text { municación e Información }\end{array}$ \\
\hline $\begin{array}{l}\text { Justis, O., Almestro, S. } \\
\text { y Silva, O. (2017) }\end{array}$ & $\begin{array}{l}\text { Pedagogía para el desarrollo socioemocio- } \\
\text { nal. A propósito de la gestión de aprendiza- } \\
\text { je en el contexto santiaguero. }\end{array}$ & $\begin{array}{l}\text { Revista Iberoamericana de } \\
\text { Educación. }\end{array}$ \\
\hline $\begin{array}{l}\text { Cuadra, D. J., Salgado, } \\
\text { J. A., Lería, F. J., \& Me- } \\
\text { nares, N. D. (2018). }\end{array}$ & $\begin{array}{l}\text { Teorías subjetivas en docentes sobre el } \\
\text { aprendizaje y desarrollo socioemocional: } \\
\text { Un estudio de caso. }\end{array}$ & \\
\hline Torres, E. (2018). & $\begin{array}{l}\text { Competencias socioemocionales y creencias } \\
\text { de autoeficacia como predictores del bur- } \\
\text { nout en docentes mexicanos. }\end{array}$ & $\begin{array}{l}\text { REXE. Revista de Estudios y } \\
\text { Experiencias en Educación. }\end{array}$ \\
\hline García, B. (2018). & $\begin{array}{l}\text { Las HSE, no cognitivas o "blandas": apro- } \\
\text { ximaciones a su evaluación. }\end{array}$ & $\begin{array}{l}\text { Revista Digital Universitaria } \\
\text { (RDU). }\end{array}$ \\
\hline $\begin{array}{l}\text { Rubiales, J., Russo, D., } \\
\text { Paneiva, J. P. y Gonzá- } \\
\text { lez, R. (2018). }\end{array}$ & $\begin{array}{l}\text { Revisión sistemática sobre los programas de } \\
\text { Entrenamiento Socioemocional para niños } \\
\text { y adolescentes de } 6 \text { a } 18 \text { años publicados en- } \\
\text { tre } 2011 \text { y } 2015 \text {. }\end{array}$ & $\begin{array}{l}\text { Revista Costarricense de Psi- } \\
\text { cología. }\end{array}$ \\
\hline $\begin{array}{l}\text { Gutiérrez, A. M. y Bui- } \\
\text { trago, S. J. (2019) }\end{array}$ & $\begin{array}{l}\text { Las HSE en los docentes: herramientas de } \\
\text { paz en la escuela. }\end{array}$ & Saber. \\
\hline $\begin{array}{l}\text { Aristulle, P. y Paoloni, } \\
\text { P. V. (2019) }\end{array}$ & $\begin{array}{l}\text { HSE en las comunidades educativas: apor- } \\
\text { tes para la formación integral de los y las } \\
\text { docentes. }\end{array}$ & ón. \\
\hline $\begin{array}{l}\text { Benítez, M. G. y Victo- } \\
\text { rino, L. (2019) }\end{array}$ & $\begin{array}{l}\text { Las HSE en la escuela secundaria mexica- } \\
\text { na: retos e incertidumbres. }\end{array}$ & $\begin{array}{l}\text { Revista Electrónica en Educa- } \\
\text { ción y Pedagogía. }\end{array}$ \\
\hline $\begin{array}{l}\text { Treviño, D. G., Gonzá- } \\
\text { lez, M. A. y Montema- } \\
\text { yor, K. M. (2019) }\end{array}$ & $\begin{array}{l}\text { HSE y su relación con el logro educativo en } \\
\text { alumnos de Educación Media Superior. }\end{array}$ & $\begin{array}{l}\text { Revista de Psicología y Cien- } \\
\text { cias del Comportamiento de la } \\
\text { Unidad Académica de Cien- } \\
\text { cias Jurídicas y Sociales: }\end{array}$ \\
\hline $\begin{array}{l}\text { Ibáñez, N. y Romero, } \\
\text { C. (2019) }\end{array}$ & $\begin{array}{l}\text { Promoviendo la competencia socioemocio- } \\
\text { nal en la infancia temprana. Estudio de ca- } \\
\text { sos. }\end{array}$ & $\begin{array}{l}\text { Cuestiones pedagógicas: Re- } \\
\text { vista de ciencias de la educa- } \\
\text { ción. }\end{array}$ \\
\hline $\begin{array}{l}\text { Sidera, F., Rostan, C., } \\
\text { Collell, J., \& Agell, S. } \\
\text { (2019). }\end{array}$ & $\begin{array}{l}\text { Aplicación de un programa de aprendiza- } \\
\text { je socioemocional y moral para mejorar la } \\
\text { convivencia en educación secundaria. }\end{array}$ & Universitas psychologica. \\
\hline
\end{tabular}




\begin{tabular}{|c|c|c|}
\hline $\operatorname{Autor}(e s)$ y año & Título & Revista indexada \\
\hline $\begin{array}{l}\text { García, L., Iriarte, C., } \\
\text { \& Reparaz, Ch. (2019). }\end{array}$ & $\begin{array}{l}\text { Apego y competencias socioemociona- } \\
\text { les del profesorado. Estado de la cuestión } \\
2015-2019 \text {. }\end{array}$ & $\begin{array}{l}\text { Revista de Psicología INFAD } \\
\text { International. }\end{array}$ \\
\hline $\begin{array}{l}\text { Castellanos, J. G. Niño, } \\
\text { S. A. y Parra, K. L. } \\
(2020) \text {. }\end{array}$ & $\begin{array}{l}\text { Discurso socioemocional y construcción } \\
\text { compartida del conocimiento en tareas co- } \\
\text { laborativas en línea. }\end{array}$ & Revista Electrónica Educare. \\
\hline
\end{tabular}

Fuente: Elaboración propia.

Los resultados de la Tabla 1 muestran que, la documentación priorizada presenta una secuencialidad lógica que comprenden producciones publicadas desde el 2016 al 2020; la estructura de los títulos articula las HSE con: La danza, formación integral de docentes y alumnos, gestión del aprendizaje, teorías subjetivas, autoeficacia, burnout, la construcción de la paz en la escuela, logros educativos, convivencia, el apego, y el desarrollo de tareas interactivas y colaborativas. Asimismo, las revistas están relacionadas con la educación, ciencias de la comunicación, el aspecto pedagógico y psicológico, y su acceso es factible en las bases de datos: SciELO, Dialnet, Google Scholar y Redalyc. La mayoría son experiencias investigativas que tienen como escenario geográfico universidades de España (5) y México (5), Argentina (2) y en mínima proporción Cuba, Chile y Colombia, y el campo de acción e intervención han sido escuelas que ofrecen el servicio educativo inicial, primaria, secundaria; y algunos programas de formación superior.

Tabla 2

Descriptores bibliográficos: Metodologías y resultados

\begin{tabular}{|c|c|c|c|}
\hline $\begin{array}{c}\text { Tipo y diseño de } \\
\text { estudio }\end{array}$ & $\begin{array}{c}\text { Población y } \\
\text { muestra }\end{array}$ & $\begin{array}{c}\text { Técnicas e } \\
\text { instrumentos }\end{array}$ & Resultados \\
\hline Revisión teórica & Temas de interés & $\begin{array}{l}\text { Análisis de } \\
\text { contenido }\end{array}$ & $\begin{array}{l}\text { Revisión teórica de la inteligencia emo- } \\
\text { cional y socioemocional }\end{array}$ \\
\hline $\begin{array}{l}\text { Revisión } \\
\text { bibliográfica }\end{array}$ & Temas de interés & $\begin{array}{l}\text { Análisis de } \\
\text { contenido }\end{array}$ & $\begin{array}{l}\text { Implementar programas socioemocio- } \\
\text { nales en la educación no formal, dise- } \\
\text { ñados en base a la neuroplasticidad y el } \\
\text { método experimental. }\end{array}$ \\
\hline $\begin{array}{l}\text { Estudio } \\
\text { etnográfico y } \\
\text { bibliográfico }\end{array}$ & $\begin{array}{l}\text { P: } 240 \text { docentes } \\
\text { M: } 80 \text { docente } \\
\text { (muestreo } \\
\text { intencional) }\end{array}$ & $\begin{array}{l}\text { Entrevistas, } \\
\text { observación } \\
\text { participante } \\
\text { grupos focales y de } \\
\text { discusión }\end{array}$ & $\begin{array}{l}\text { Identifica aspectos relacionados con } \\
\text { una educación tradicional centrada en } \\
\text { el docente, practica de actitudes inade- } \\
\text { cuadas para autorregular la disciplina, } \\
\text { y decisiones autoritarias en la solución } \\
\text { de conflictos. }\end{array}$ \\
\hline
\end{tabular}




\begin{tabular}{|c|c|c|c|}
\hline $\begin{array}{c}\text { Tipo y diseño de } \\
\text { estudio }\end{array}$ & $\begin{array}{c}\text { Población y } \\
\text { muestra }\end{array}$ & $\begin{array}{c}\text { Técnicas e } \\
\text { instrumentos }\end{array}$ & Resultados \\
\hline $\begin{array}{l}\text { Metodología } \\
\text { cualitativa }\end{array}$ & \multirow{3}{*}{$\begin{array}{l}19 \text { docentes } \\
\text { (muestreo } \\
\text { intencional de tipo } \\
\text { opinático) }\end{array}$} & $\begin{array}{l}\text { Entrevistas } \\
\text { episódicas }\end{array}$ & \multirow{3}{*}{$\begin{array}{l}\text { Analiza e interpreta las teorías subjeti- } \\
\text { vas en relación al sentido del aprendi- } \\
\text { zaje y el desarrollo socioemocional. }\end{array}$} \\
\hline $\begin{array}{l}\text { Estudio descriptivo } \\
\text { interpretativo }\end{array}$ & & $\begin{array}{l}\text { Grupos de } \\
\text { discusión }\end{array}$ & \\
\hline Estudio de caso & & $\begin{array}{l}\text { Análisis de } \\
\text { contenido }\end{array}$ & \\
\hline $\begin{array}{l}\text { Tipo descriptivo } \\
\text { Diseño no } \\
\text { experimental }\end{array}$ & $\begin{array}{l}\text { PM: } 549 \text { docentes } \\
\text { (muestreo no } \\
\text { probabilístico) }\end{array}$ & $\begin{array}{l}\text { Inventarios y } \\
\text { escalas. }\end{array}$ & $\begin{array}{l}\text { El análisis del Burnout indica que la } \\
\text { despersonalización es lo que más se re- } \\
\text { fleja, seguido del agotamiento emocio- } \\
\text { nal y la insatisfacción de logro. }\end{array}$ \\
\hline $\begin{array}{l}\text { Revisión y análisis } \\
\text { bibliográfico }\end{array}$ & Programas & $\begin{array}{l}\text { Análisis de } \\
\text { contenido }\end{array}$ & $\begin{array}{l}\text { Describe habilidades no cognitivas y } \\
\text { competencias socioemocionales, }\end{array}$ \\
\hline $\begin{array}{l}\text { Revisión } \\
\text { sistemática }\end{array}$ & $\begin{array}{l}19 \text { artículos } \\
\text { seleccionados }\end{array}$ & $\begin{array}{l}\text { Formato de } \\
\text { revisión }\end{array}$ & $\begin{array}{l}\text { Revisión de } 19 \text { artículos publicados en- } \\
\text { tre los años } 2011-2015 .\end{array}$ \\
\hline Revisión teórica & Temas de interés & $\begin{array}{l}\text { Análisis de } \\
\text { contenido }\end{array}$ & $\begin{array}{l}\text { Revisión de contenidos teóricos rela- } \\
\text { cionados con: La escuela, el conflicto } \\
\text { y la paz en escenarios formativos y de } \\
\text { socialización; }\end{array}$ \\
\hline $\begin{array}{l}\text { Tipo descriptivo } \\
\text { Diseño no } \\
\text { experimental }\end{array}$ & $\begin{array}{l}\text { P: Todos los } \\
\text { estudiantes. } \\
\text { M: } 106 \\
\text { estudiantes. } \\
\text { (muestreo } \\
\text { intencional) }\end{array}$ & $\begin{array}{l}\text { Cuestionario } \\
\text { sobre habilidades } \\
\text { Percibidas }\end{array}$ & $\begin{array}{l}\text { El } 47,1 \% \text { de los participantes presentan } \\
\text { escaso desarrollo de la habilidad para } \\
\text { tomar conciencia de los sentimientos } \\
\text { que experimentan; } 75 \% \text { y } 80 \% \text { mues- } \\
\text { tran dificultades para organizarse en } \\
\text { grupos; y un } 60 \% \text { tienen poco desarro- } \\
\text { lladas las habilidades para perseverar y } \\
\text { tolerar las presiones estresantes. }\end{array}$ \\
\hline $\begin{array}{l}\text { Metodología } \\
\text { cualitativa } \\
\text { Análisis de } \\
\text { documentos. }\end{array}$ & $\begin{array}{l}\text { Políticas, leyes y } \\
\text { modelo educativo }\end{array}$ & $\begin{array}{l}\text { Análisis de } \\
\text { contenido }\end{array}$ & $\begin{array}{l}\text { Análisis y reflexión sobre la percepción } \\
\text { de la vida emocional en el escenario } \\
\text { actual }\end{array}$ \\
\hline $\begin{array}{l}\text { Enfoque } \\
\text { cuantitativo } \\
\text { Tipo no } \\
\text { experimental }\end{array}$ & 4822 estudiantes & $\begin{array}{l}\text { Cuestionario de } \\
\text { contexto }\end{array}$ & $\begin{array}{l}\text { La toma de decisiones positivas tuvo } \\
\text { mayor relación con los logros educati- } \\
\text { vos, menor relación con la perseveran- } \\
\text { cia y la autorregulación, además pre- } \\
\text { senta una asociación significativa con } \\
\text { la empatía. }\end{array}$ \\
\hline $\begin{array}{l}\text { Enfoque } \\
\text { cualitativo }\end{array}$ & \multirow{3}{*}{$\begin{array}{l}4 \text { docentes } \\
\text { (muestreo dirigido } \\
\text { - homogéneo) }\end{array}$} & $\begin{array}{l}\text { Entrevista no } \\
\text { estructurada }\end{array}$ & \multirow{3}{*}{$\begin{array}{l}\text { Análisis comparativo de dos programas } \\
\text { (9M9E y EDE) orientados al desarrollo } \\
\text { de la competencia socioemocional en } \\
\text { educación infantil. }\end{array}$} \\
\hline $\begin{array}{l}\text { Metodología } \\
\text { inductiva }\end{array}$ & & $\begin{array}{l}\text { Análisis } \\
\text { documental }\end{array}$ & \\
\hline Estudio de casos & & & \\
\hline
\end{tabular}




\begin{tabular}{|c|c|c|c|}
\hline $\begin{array}{l}\text { Tipo y diseño de } \\
\text { estudio }\end{array}$ & $\begin{array}{c}\text { Población y } \\
\text { muestra }\end{array}$ & $\begin{array}{c}\text { Técnicas e } \\
\text { instrumentos }\end{array}$ & Resultados \\
\hline $\begin{array}{l}\text { Enfoque } \\
\text { cuantitativo } \\
\text { Tipo } \\
\text { experimental: } \\
\text { Correlacional }\end{array}$ & 64 participantes & $\begin{array}{l}\text { Test y } \\
\text { cuestionarios }\end{array}$ & $\begin{array}{l}\text { El análisis estadístico muestra que el } \\
\text { programa de intervención basado en } \\
11 \text { sesiones, no generó cambios signi- } \\
\text { ficativos en la convivencia estudiantil. }\end{array}$ \\
\hline $\begin{array}{l}\text { Revisión del } \\
\text { estado de la } \\
\text { cuestión. }\end{array}$ & $\begin{array}{l}22 \text { artículos } \\
\text { publicados. }\end{array}$ & $\begin{array}{l}\text { Análisis de } \\
\text { contenido }\end{array}$ & $\begin{array}{l}\text { Revisión de } 22 \text { estudios publicados en- } \\
\text { tre los años } 2015 \text { y } 2019 .\end{array}$ \\
\hline $\begin{array}{l}\text { Estudio cualitativo } \\
\text { Casos múltiples }\end{array}$ & $\begin{array}{l}\text { Muestra de } \\
\text { trabajo: Tres } \\
\text { grupos de } 5 \\
\text { estudiantes. }\end{array}$ & $\begin{array}{l}\text { Análisis de } \\
\text { contenido }\end{array}$ & $\begin{array}{l}\text { El tipo de discurso socioemocional con } \\
\text { mayor frecuencia en la cadena socio- } \\
\text { cognitiva es el afectivo, valorativo y de } \\
\text { apoyo. }\end{array}$ \\
\hline
\end{tabular}

La Tabla 2 indica que, los tipos de investigación implementados se corresponden con metodologías cualitativas y cuantitativas, y entre los diseños utilizados se encuentran: Revisión teórica, bibliográfica, analítica y sistemática, de igual forma, los estudios descriptivos, interpretativos, correlacionales, de casos, etnográficos e inductivos. La población y muestra que fueron seleccionadas estuvieron representadas por docentes, estudiantes, programas y artículos. Y, las técnicas e instrumentos de gabinete y de campo fueron: entrevistas, observaciones participativas, modelación, grupos focales y de discusión, inventarios, escalas, formatos de revisión, cuestionarios, test y análisis de contenido. En contraste, afirmar que predominan indagaciones de revisión teórica, escaso involucramientos de la comunidad educativa, e insuficientes investigaciones emprendidas y divulgadas por maestros que ejercen su labor educativa en instituciones educativas.

En lo referente a los resultados de las investigaciones revisadas, Aragó, Zorrilla y Balaguer (2016) realiza un análisis histórico - teórico de diversas conceptualizaciones como la inteligencia emo- cional y socio emocional, educación emocional, y aborda la danza como una estrategia para el aprendizaje socioemocional en los niños y niñas, y en base a esta revisión elabora una propuesta metodológica para trabajar las HSE mediante el ejercicio de la danza en educación infantil. Por su parte, Jakiwara (2016) luego de haber examinado bibliografía especializada y tener conocimiento de investigaciones sobre HSE propone como resultados contextualizar programas de habilidades sociales y emocionales e incluirlos en la educación no formal, es decir adaptarlos a entornos extraescolares, asimismo, hacer uso de la investigación acción, métodos experimentales, esbozar el enfoque de la neuroplasticidad y desarrollar talleres donde se traten tópicos coherentes con la formación integral humana.

Justis, Almestro y Silva (2017) determinan sus resultados a partir del análisis, comprensión e interpretación etnográfica que realizan al pensamiento pedagógico docente en relación a las HSE en dos institutos, encuentran brechas entre las actuaciones del egresado con el contexto laboral, especialmente identifican una insuficiente 
formación en el manejo emocional y el uso de procedimientos inadecuados para interactuar en las aulas, imperando por ende un ejercicio docente tradicional; esta realidad también se percibe en los directivos y en la atención a los padres de familia. Frente a ello, construyen conceptualizaciones colectivas que conlleve a una pedagogía socioemocional. La estructura de esta iniciativa comprende procesos dialécticos y de revisión crítica de espacios socioeducativos, y se sistematiza en diadas lógicas: Requisitos - premisas, y Social - Escolar.

Cuadra et al., (2018) los resultados que exponen indican que, el aprendizaje y desarrollo socioemocional se configuran como dimensiones transversales del currículo, por tanto, la enseñanza debe enfocarse en el reconocimiento, expresión y regulación de emociones y garantizar el bienestar y el sentido a la vida y el aprendizaje. Los factores que se consideran e intervienen en este proceso están representados en lo socioeconómico y cultural, las características individuales, el rol de la familia y los ambientes escolares. Asimismo, determinan que, el desarrollo socioemocional está sujeto a etapas evolutivas: inmadurez, inicios de la madurez, crisis, y madurez socioemocional. También establecen propuestas formativas como la educación parental, el uso de estrategias pedagógicas, y el diseño de macro y micropolíticas en favor de la educación socioemocional. Finalmente reconocen la importancia de promover la formación socioemocional en docentes y estudiantes.

Torres (2018) presenta como hallazgos significativos: Las competencias socioemocionales de mayor dominio por parte de los maestros es la conciencia emocional y su espíritu optimista, seguido de las actitudes empáticas y el manejo autónomo de sus emociones. En relación con las creencias de autoeficacia la percepción que predomina es el interés por construir un clima de aula favorable para la interacción y el aprendizaje; no obstante, los indicadores referidos a la implicación del estudiante en el aprendizaje y la evaluación tiene una percepción desfavorable y de poca relevancia por cuestionamientos y controversias. Y los resultados diagnósticos que consiguieron de la Escala de Desgaste Ocupacional expresan que, los docentes exteriorizan un alto nivel de despersonalización (indolencia o cinismo) sobre todo en sus relaciones y actuaciones laborales, igualmente muestran desgaste personal, emocional y profesional; así como insatisfacción de su desempeño y logros.

A su vez García (2018), describe e interpreta diversos aportes teóricos concatenados a las HSE, permitiéndole identificar aspectos esenciales impregnados en el núcleo de las HSE (agencia, identidad integrada y competencias) los mismos que son claves para el éxito en la temprana adultez; señalan también la perseverancia, autorregulación y trabajo en equipo como habilidades básicas en la motivación, ser conscientes de nuestras emociones, pensamientos y acciones, y tomar decisiones colaborativas. Agrega además que, en el contexto educativo, laboral y personal es importante el desarrollo de habilidades inter e intrapersonales en armonía con las habilidades cognitivas. Por otro lado, expresa percepciones sobre la evaluación de las HSE y los instrumentos que se utilizan, manifestando que existen ciertos sesgos de referencia, persistencia en la aplicación de cuestionarios, test e informes tradicionales, y plantea la necesidad de cumplir con estándares de calidad a través de procedimientos psicométricos sólidos y rigurosos de validez y confiabilidad.

Rubiales et al., (2018) revisaron y analizaron programas de entrenamiento socioemocional, en- 
contrando que, la mayoría de estudios fueron desarrollados en España, y los objetivos de mayor frecuencia se centraron en la inteligencia emocional, la modalidad de intervención fue grupal; utilizaron recursos orales y escritos, la modelación se hizo en el aula y la responsabilidad de su aplicación estuvieron a cargo de docentes y psicólogos; a su vez, la metodología se manifestó en procesos experimentales, participaron niños, niñas y adolescentes, y los tiempos asignados de dos a cuatro semanas. Describe y sintetiza los programas resaltando los efectos positivos en la mejora significativa de variables referidas a: competencia social y emocional, inteligencia socio - emocional; percepción, facilitación y regulación emocional; salud mental, autoconcepto, empatía, autoestima, realización social, autoeficacia, rendimiento académico, locus de control; adiciona, la disminución de la ansiedad, depresión, problemas socioemocionales, agresividad y el comportamiento antisocial.

Gutiérrez y Buitrago (2019) exploran e interpretan conocimientos respecto a la incidencia de las HSE de los docentes como herramientas de gestión en la construcción de ambientes acogedores y de paz. Explican que la escuela es un espacio de formación y socialización y tiene la función de cimentar los valores, actitudes, habilidades de los estudiantes y con ello prevenir todo tipo de violencia y conflictos culturales; complementan al señalar la importancia del aprendizaje social y emocional en el clima de aula, las relaciones e interacción profesor-alumnos, alumno-alumno y motivación escolar; asimismo, plantean que, la transversalidad de las HSE dentro del currículo permite convivir y coexistir en sociedad. Reconocen también que, la práctica de las HSE juega un rol esencial en los procesos de enseñanza aprendizaje, además, favorece el bienestar de los maestros; sin embargo, resaltan la necesidad de fortalecer las HSE en los profesores para integrar y articular la educación básica y la educación inclusiva.

Aristulle y Paoloni (2019) luego de aplicar y procesar el cuestionario de Habilidades Percibidas muestran los siguientes resultados: Los estudiantes de primer, tercer y cuarto año de la especialidad primaria e inicial tienen escasa conciencia de sus sentimientos que expresan $(47,1 \%)$, limitaciones para integrarse, liderar y trabajar en equipo $(75 \%$ y $80 \%)$, así como perseverar ante la adversidad, tolerar presiones y situaciones de estrés (50\% y 60\%). Sin embargo, son empáticos y se vinculan fácilmente con sus pares (62\% y $64 \%)$; son capaces de autocontrolar sus impulsos para alcanzar metas (49\%); asimismo, manifiestan optimismo y un estado de ánimo positivo a pesar de las condiciones adversas (80\%). Y, la mayoría están seguros de sí mismo y pueden adaptarse a los cambios o situaciones inciertas $(60 \%)$. Frente a estos resultados proponen reorientar los diseños curriculares y garantizar el desarrollo de las HSE en la formación de los futuros maestros.

Benítez y Victorino (2019) organizan los resultados cualitativos en cuatro temas de análisis. En el primer tema, perciben la vida emocional en un contexto de constantes cambios de las condiciones humanas generada por una sociedad líquida, los sistemas económicos y modos de producción; y las crisis psicológicas en sus diferentes formas como la inhibición, malestar y pobreza emocional e incremento de las emociones negativas y la irracionalidad de la conciencia. En el segundo tema, valoran las HSE como herramientas que ayudan a comprender, regular y manejar las emociones responsablemente y asumen que las competencias emocionales son capacidades que movilizan conocimientos, actitudes y habilidades en la consecución de actividades de aprendiza- 
je. En el tercer tema, determinan que, la escuela siempre ha sido un espacio donde se han puesto de manifiesto las emociones, afectos y sentimientos. Y, en el cuarto tema, analizan el área de tutoría y educación emocional, describiendo el tiempo asignado para su desarrollo y las dimensiones que son parte de su estructura (autoconocimiento, autorregulación, autonomía, empatía y colaboración). Por último, hacen referencia a retos e incertidumbres a tener en cuenta en la inclusión y transversalidad de la HSE en el nivel secundario, entre ellos: Formación de los docentes en el dominio de las habilidades y competencias emocionales, y reconfigurar el rol de los tutores.

Treviño et al., (2019), presentan resultados generados por el análisis factorial de las evaluaciones del 2017 en estudiantes de Educación Media Superior de México. Comprobando que, las relaciones de los factores (perseverancia, empatía, autorregulación, toma de decisiones positivas y negativas) fueron significativas y aceptables con los logros educativos. Identifican que el factor expresado en tomar decisiones positivas tiene mayor peso en el aprendizaje, luego se encuentra la perseverancia; en un término medio o estándar se sitúan la empatía y la autorregulación. En el análisis del modelaje relacional determinan que las decisiones negativas están conectadas a la perseverancia y a una baja autorregulación; no obstante, los demás factores socioemocionales se complementan e interaccionan positiva y significativamente en el avance progresivo del rendimiento académico.

Ibáñez y Romero (2019), analizan y comparan dos programas de educación emocional en el nivel preescolar, evidenciando que ambos modelos presentan coincidencias y diferencias. Coinciden en: desarrollar la educación emocional desde la etapa infantil, para tal fin enfocan sus esfuerzos en la formación, gestión de recursos y materiales, y la participación de los padres de familia. Asimismo, en ambos programas, perciben cambios sustanciales en las relaciones interpersonales, los niños y niñas son capaces de reconocer, gestionar, expresar emociones en forma oral y gestual; además, mejoraron la autoestima, comportamiento, el afecto mutuo, redujeron las situaciones conflictivas y agresivas entre ellos, esto último conllevó a sostener un clima de aula atractivo y pacífico. Así también, estas propuestas beneficiaron a los maestros en lo personal y profesional: en lo primero aumentó la empatía y el conocimiento de sí mismo; y en lo segundo, favoreció la sinergia de iniciativas para innovar y diseñar las propuestas.

Sidera et al., (2019) aplican 11 sesiones tutoriales con el propósito de mejorar la convivencia en alumnos de secundaria, y los resultados obtenidos tanto en el pre y post test confirman que, en los grupos (control y experimental) las conductas agresivas premeditadas e impulsivas se mantienen, la empatía se incrementa en los sujetos de estudio, pero no es significativo su cambio, de manera similar ocurre con el clima y la desconexión moral (autoconcepto negativo). Por consiguiente, desde el análisis estadístico el programa de aprendizaje socioemocional que ejecutaron no generó mejoras sustantivas en las formas de actuar y convivir de los estudiantes.

En tanto, García et al., (2019) procesan información sobre el apego escolar en diversas publicaciones, constatando resultados relevantes que se denotan en la relación profesor - estudiante. Confirmando que este vínculo favorece al alumno internalizar y transferir aprendizajes, fomenta el desarrollo socioemocional y la práctica de habilidades sociales, permite el ajuste emocional de los niños, mejora el desempeño y la enseñanza de los docentes. Mencionan además que el apego no es beneficioso con los comportamientos inade- 
cuados, y sugieren investigar tipologías del apego que cultiven y aseguren la calidad de las buenas relaciones entre docente y alumno.

Castellanos et al., (2020) exploran diálogos interactivos y emocionales que manifiestan los estudiantes en foros grupales asincrónicos, y obtienen como resultado lo siguiente: frecuente práctica de discursos que cohesionan el trabajo colaborativo (expresiones de cordialidad y uso de pronombres inclusivos), discursos para mantener un estado de ánimo positivo, discursos expresados en el apoyo mutuo y los buenos deseos e interés para desarrollar la tarea; así también, resaltan los discursos que valoran los esfuerzos colaborativos, y motivan el progreso de las tareas. Señalan que, todos estos discursos contribuyen en la efectividad de las fases de las cadenas sociocognitivas: Organizar tareas, diagnosticar el tema, construir significados, sistematizar aprendizajes y llegar a acuerdos. Por tanto, los discursos socioemocionales recurrentes en actividades digitales benefician compartir experiencias, aprendizajes y la construcción de conocimientos colaborativos.

\section{Discusión}

El propósito del estudio se centró en la revisión de información válida y confiable de las HSE en las instituciones educativas. En ese sentido se realizó un análisis descriptivo, sistemático y riguroso de los principales aportes que presentan los artículos priorizados. La revisión de las investigaciones científicas confirman que las habilidades socioemocionales tienen mayor desarrollo en instituciones educativas españolas y mexicanas. Estos conocimientos válidos y confiables están publicados en revistas indexadas educativas, pedagógicas y psicológicas y vinculados a las bibliotecas web Google Scholar SciELO, Redalyc y
Dialnet; por su parte, los procesos metodológicos utilizado son los enfoques cualitativos y cuantitativo, los sujetos que participan en los estudios comprenden docentes, estudiantes y comunidades educativas, y las técnicas e instrumentos más recurrentes fueron la revisión documental, entrevista, observación, encuestas, inventarios, matriz de análisis; Asimismo, las producciones documentadas corroboran el impacto positivo y efectivo de las HSE en la gestión del aprendizaje, las emociones y los conflictos, mejoras del trabajo de equipo, las relaciones y comunicación interpersonales, y sienta las bases de una cultura de paz.

Los hallazgos diagnósticos muestran percepciones objetivas y apreciaciones subjetivas que justifican el desarrollo e implementación de las HSE en la educación básica. Justis et al., (2017) revelan que sigue persistiendo una educación instructiva centrada en el protagonismo del docente, formas verticalistas para controlar el comportamiento de los estudiantes y decisiones autocríticas de directores y docentes en la solución de situaciones conflictivas institucionales; Aristulle y Paoloni (2019) complementan al afirmar que la mayoría de estudiantes expresa sus sentimientos de manera inconsciente $(47,1 \%)$, limitaciones para integrarse en equipos de trabajo (80\%), afrontar y superar con firmeza frustraciones, adversidades y presiones (60\%); y, Torres (2018) agrega el problema del Burnout que se manifiesta en los docentes, el mismo que viene generando el agotamiento o estrés emocional, la insatisfacción del trabajo que realiza, y la despersonalización es decir muchos maestros prefieren aislarse, desconectarse de la realidad, asumir actitudes conformistas, evadir responsabilidades y actuar con cinismo en sus interacciones.

No obstante, frente a esta realidad, para muchos investigadores las HSE constituyen herramientas 
que van a contribuir en mejorar los procesos de enseñanza aprendizaje y fortalecer la formación emocional de los docentes. Gutiérrez y Buitrago (2019) resaltan su influencia en el bienestar y la relación de los actores educativos, la práctica de valores, gestión de ambientes escolares pacíficos, socialización e inclusión formativa, y desarrollo de conductas prosociales; García et al., (2019) por su parte, destacan los beneficios en la construcción del apego afectivo y armónico entre los docentes y estudiantes, el mismo que trae consigo incremento en el progreso del aprendizaje y mejora del desempeño pedagógico. Y, Aristulle y Paoloni (2019) desde su análisis diagnóstico nos dice que las HSE, desarrolla la empatía (62\%), potencia los vínculos estudiantiles (64\%), autorregulan sus impulsos para alcanzar metas (49\%) estimula estados de ánimo positivos (80\%), y ayuda en la planificación de actuaciones y adaptaciones coherentes en entornos cambiantes. Desde estas perspectivas, se corrobora aportes significativos de las HSE en las organizaciones escolares.

Con base a este análisis contrastivo, la configuración pedagógica, dialéctica y holística de las HSE se fundamenta en los principios y postulados de las teorías: inteligencia emocional de Goleman, inteligencias múltiples de Gardner (1983) y el aprendizaje social de Bandura (1987); estos enfoques orientan procesos educativos encauzados en la formación socioemocional de estudiantes y docentes como contrapeso de la formación cognitiva, que busca asegurar la integralidad del ser humano. También, se sustenta en modelos teóricos emergentes como las habilidades sociales, neuroplasticidad, educación emocional, pedagogía de las emociones, etc., que aportan contenidos pertinentes y funcionales para su implementación en los centros educativos; sin embargo, a pesar de esta diversidad de planteamientos, aún existen ciertos vacíos en cuanto a su delimitación conceptual, cuerpo teórico y metodología del campo de acción.

Con respecto al entrenamiento e intervención de las HSE, Aragó et al., (2016) proponen abordar pedagógicamente la danza como una estrategia recreativa para el desarrollo y aprendizaje socioemocional, Jakiwara (2016) establece aplicar programas de HSE en ámbitos extraescolares o informales, y recomienda hacer uso de metodologías cualitativas y responder a la realidad socioemocional de los sujetos; Justis et al., (2017) diseñan una matriz dialéctica que guía la planificación e intervención de acciones dirigidas al desarrollo socioemocional y la gestión del aprendizaje, en esta misma línea de análisis, Ibáñez y Romero (2019) luego de revisar dos proyectos socioemocionales confirman impactos positivos en la gestión de la emociones y mejora de las relaciones interpersonales, así como también en los docentes dominio de sus emociones en el plano personal, familiar y profesional. En contraste, Sidera et al., (2019) aseveran que el programa tutorial que aplicaron tuvo resultados desfavorables, por tanto, discrepan de los efectos que puedan generar las HSE en la convivencia y disminución de la agresividad.

Mientras Castellanos et al., (2020) exponen la importancia de los discursos socioemocionales en la construcción de conocimientos y aprendizajes colaborativos e interactivos; Treviño et al., (2019) añaden que, los estudiantes para alcanzar logros educativos deben ser capaces de tomar decisiones positivas, ser perseverantes, empáticos, y saber autorregularse. Y, Cuadra et al., (2018) desde su interpretación de teorías subjetivas concluye que las HSE tienen relación con el sentido de la vida, su vivencia y práctica depende en cierta forma de factores sociales, familiares, personales, escolares; igualmente su desarrollo evolutivo 
comprende estadios, y que el abordaje las HSE en las instituciones educativas es un desafío complejo que necesita de estrategias pedagógicas y la formación de los estudiantes y profesorado en el manejo de sus competencias socioemocionales.

Por consiguiente, las experiencias de intervención muestran significativas contribuciones de las habilidades socioemocionales en la mejora de los aprendizajes, desarrollo de competencias emocionales y su incidencia en la construcción de un clima organizacional favorable. Las propuestas de algunos investigadores se centran en utilizar la danza como estrategia de motivación y estimulación de habilidades en educación infantil; promoción del apego afectivo entre docente alumnos; la práctica del discurso socioemocional en actividades digitales del aprendizaje; además de organizar y entrenar programas tutoriales socioemocionales; de igual modo, plantean la aplicación de matrices pedagógicas dialécticas; no obstante, reconocer que la mayoría de investigadores se enfocó en la revisión teórica, y en la evaluación del componente emocional a través de inventarios y test disociados y en muchos casos con relativa congruencia con la variable de estudio.

\section{Conclusiones}

La revisión de los estudios sobre el desarrollo de habilidades socioemocionales en las instituciones educativas se realizó mediante el ingreso a bases de datos científicas electrónicas y permitió hacer un análisis riguroso y ordenado de estudios teóricos-reflexivos, y experiencias de intervención educativa.

En las instituciones educativas, las HSE que se plantean desarrollar $-\mathrm{y}$ algunas escuelas vienen desarrollando- comprenden: Autoconciencia, autocontrol, automotivación, empatía y destreza social (Aragó et al. 2016, 159); habilidades comunicativas, asertivas, empáticas, control interactivo, predictivas y de toma de decisiones (Justis et al., 2017, 119); perseverancia, curiosidad y sociabilidad (García 2018, 4); autoconocimiento, autorregulación, autonomía, empatía y colaboración (Benítez y Victorino 2019, 138); perseverancia, empatía, autorregulación, toma de decisiones (Treviño et al., 2019). Resaltar, que estas habilidades difieren en sus percepciones y alcances contextuales, asociándolas además como competencias y aprendizajes socioemocionales.

Los aportes de la HSE en las instituciones de educación básica y superior se expresan en la mejora de la gestión de los procesos de enseñanza - aprendizajes, el clima escolar, formación integral, trabajo colaborativo presencial y virtual, fortalecimiento de los vínculos de convivencia en la comunidad educativa, bienestar, salud y sentido de vida personal e interpersonal; regulación ética y moral de actuaciones, sentimientos y emociones; autoeficacia y autonomía en el desempeño pedagógico; construcción de una personalidad y profesionalidad competente, firme, coherente, constructiva y potente frente a la adversidad; todas estas son herramientas efectivas para compartir y producir conocimientos, ofrecer un servicio de calidad, establecer relaciones auténticas y gestionar la paz.

Financiamiento

Declaración de conflicto de interés

\section{Referencias}

Aragó, A., Zorrilla, L. y Balaguer, P. (2016). Como trabajar las habilidades socioemocio- 
nales a través de la danza y su importancia en la escuela. Quaderns digitals: Revista de Nuevas Tecnologías y Sociedad, ISSN-e 1575-9393, Nº 82, 2016, págs. 155-172. Disponible en: file:///C:/ Users/USUARIO/Downloads/archivoPDF.pdf

Aristulle, P. y Paoloni P. V. (2019). Habilidades socioemocionales en las comunidades educativas: aportes para la formación integral de los y las docentes. Revista Educación. On-line versión ISSN 2215-644Print version ISSN 0379-7082. Educación vol.43 n.2 San José, San Pedro, Montes de Oca Jul./Dec. 2019. Disponible en: https:// www.scielo.sa.cr/pdf/edu/v43n2/2215-2644EDU-43-02-00049.pdf

Barrientos, A. (2016). Habilidades sociales y emocionales del profesorado de educación infantil relacionadas con la gestión del clima de aula (Tesis Doctoral, Universidad Complutense de Madrid, Madrid, España). Recuperado de https://eprints.ucm.es/40450/

Bandura, A. (1987). Teoría del aprendizaje social. Espasa-Calpe.

Benítez, M. C. y Victorino, L. (2019). Las habilidades socioemocionales en la escuela secundaria mexicana: retos e incertidumbres. Revista Electrónica en Educación y Pedagogía, 3 (5), 129-144. [Fecha de Consulta 26 de agosto de 2020]. ISSN: Disponible en: https://www.redalyc.org/articulo. oa?id=5739/573962080013

Castellanos, J. C., Niño, S. A. y Parra, K. L. (2020). Discurso socioemocional y construcción compartida del conocimiento en tareas colaborativas en línea. Revista Electrónica Educare, 24 (2), 59-79. [Fecha de Consulta 26 de agosto de 2020]. ISSN: Disponible en: https://www.redalyc.org $/$ articulo.oa?id=1941 $/ 194163269004$

Cerezal, J. y Fiallo, J. (2002). Los métodos científicos en las investigaciones pedagógicas (pp. 45-61).
Guadra, D. J., Salgado, J. A., Lería, F. J., \& Menares, N. D. (2018). Teorías subjetivas en docentes sobre el aprendizaje y desarrollo socioemocional: Un estudio de caso. Revista Educación, 42 (2), 1-33. [Fecha de Consulta 26 de agosto de 2020]. ISSN: 0379-7082. Disponible en: https://www.redalyc. org/articulo.oa?id=440/44055139014

Del Rey, R. y Ortega, R. (2001). El Programa de ayuda entre iguales en el contexto del Proyecto Sevilla Antiviolencia Escolar. Revista de Educación, 326, 197-310.

García, B. (2018). Las habilidades socioemocionales, no cognitivas o "blandas": aproximaciones a su evaluación. Revista Digital Universitaria (RDU). Vol. 19, núm. 6 noviembre-diciembre. Doi: http://doi.org/10.22201/ codeic.16076079e.2018.v19n6.a5.

García, L., Iriarte, C, \& Reparaz, Ch. (2019). Apego y competencias socioemocionales del profesorado. Estado de la cuestión 20152019. Revista Internacional de Psicología del Desarrollo y la Educación, 2 (1), 119-128. [Fecha de Consulta 26 de agosto de 2020]. ISSN: 0214-9877. Disponible en: https://www.redalyc.org/articulo. oa?id=3498/34986012601 1

Gardner, (1983). Multiple Intelligences, ISBN 0-465-04768-8, Basic Books. Castellano "Inteligencias múltiples” ISBN: 84-493-1806-8 Paidos.

Goleman, D. (1995). Inteligencia Emocional. Estados Unidos: Editorial Bantam Books.

Gutiérrez, A. M. y Buitrago, S. J. (2019). Las habilidades socioemocionales en los docentes: herramientas de paz en la escuela. Praxis $\mathcal{E}^{\circ}$ Saber, Print version ISSN 2216-0159. Prax. Saber vol.10 no.24 Tunja Sep./Dec. 2019. Disponible en: http://www.scielo.org.co/pdf/prasa/ v10n24/2216-0159-prasa-10-24-167.pdf 
Ibáñez, N. y Romero, C. (2019). Promoviendo la competencia socioemocional en la infancia temprana. Estudio de casos. Cuestiones pedagógicas: Revista de ciencias de la educación. ISSN 0213-1269, ISSN-e 2253-8275, No. 28, 2019, págs. 33-48. Disponible en: https://institucional.us.es/revistas/cuestiones/28/04_A03_2019.pdf

Jakiwara, L. G. (2016). Las habilidades socioemocionales en los jóvenes: una propuesta de desarrollo humano integral. Revista de Ciencias de la Comunicación e Información, 21 (2), 67-80. Disponible en: file:///C:/Users/USUARIO/Downloads $/ 44$-Texto $\% 20 \mathrm{del}^{\circ} \% 20 \mathrm{art} \% \mathrm{C} 3 \% \mathrm{ADcu}$ lo-42-2-10-20200609.pdf

Justis, O., Almestro, S. y Silva, O. (2017). Pedagogía para el desarrollo socioemocional. A propósito de la gestión de aprendizaje en el contexto santiaguero. Revista Iberoamericana de Educación. ISSN-e 1681-5653, ISSN 1022-6508, Vol. 75, $\mathrm{N}^{\circ}$. Extra 2, págs. 109-126.Disponible en: https://rieoei.org/RIE/article/view/2636/3618

Ministerio de Educación (2017). Currículo Nacional de la Educación Básica. Primera Edición. Impreso en el Perú. Lima, Perú.

Otzen, T. y Manterola, C. (2017). Técnicas de muestreo sobre una población a estudio. Int. 7. Morphol, 35(1), 227-232. https://scielo. conicyt.cl/scielo.php?script=sci_arttext\&pi$\mathrm{d}=$ S07 1795022017000100037

Romero, H. (2016). Inteligencia Emocional y Clima Organizacional. Universidad Rafael Landívar. Facultad de Humanidades. Quetzaltenango - Guatemala.

Royo, M. (2013a). Centros emocionalmente inteligentes. En P. Darder (Coord.). Aprender y educar con bienestar y empatía. La formación emocional del profesorado (pp. 45-67). Barcelona: Ediciones Octaedro.

Rubiales, J., Russo, D., Paneiva, J. P. y González, R. (2018). Revisión sistemática sobre los programas de Entrenamiento Socioemocional para niños y adolescentes de 6 a 18 años publicados entre 2011 y 2015. Revista Costarricense de Psicología, 37 (2), 163-186. [Fecha de Consulta 26 de agosto de 2020]. ISSN: 0257-1439. Disponible en: https://www.redalyc.org/articulo. oa?id $=4767 / 476759451006$

Scott, C. (2015). El futuro del aprendizaje $2{ }_{\text {¿Qué tipo }}$ de aprendizaje se necesita en el siglo XXI? Investigación y prospectiv en educación [documentos de trabajo, UNESCO]. Recuperado de: http://unesdoc. unesco.org/images/0024/002429/242996s.pdf.

Sidera, F., Rostan, C., Collell, J., \& Agell, S. (2019). Aplicación de un programa de aprendizaje socioemocional y moral para mejorar la convivencia en educación secundaria. Universitas Psychologica, 18(4), 1-14. https://doi.org/10.11144/ Javeriana.upsy18-4.apas

Torres, E. (2018). Competencias socioemocionales y creencias de autoeficacia como predictores del burnout en docentes mexicanos. REXE. Revista de Estudios y Experiencias en Educación, 17 (35), 15-27. [Fecha de Consulta 26 de agosto de 2020]. ISSN: 0717-6945. Disponible en: https://www.redalyc. org $/$ articulo.oa? id $=2431 / 243158173001$

Treviño, D. C., González, M. A. y Montemayor, K. M. (2019). Habilidades socioemocionales y su relación con el logro educativo en alumnos de Educación Media Superior. Revista de Psicología y Ciencias del Comportamiento de la Unidad Académica de Ciencias furídicas y Sociales: RPCC-UACFS. ISSN 2007-1833, Vol. 10, No. 1, 2019, págs. 3248 Disponible en: file:///C:/Users/USUARIO/ 
Downloads/Dialnet-HabilidadesSocioemocionalesYSuRelacionConElLogroEd-7054681.pdf

Vaello, J. (2009). El profesor emocionalmente competente. Un puente sobre "aulas" turbulentas. Barcelona: Editorial Graó.

West, R. M. (2016). Should non-cognitive skills be included in school accountability systems? Preliminary evidence from California's core Districts. Evidence Speaks Reports, 1, (13). 